\title{
Editorial
}

\section{New horizon in autophagy research}

\author{
Wei-dong LE ${ }^{1, *}$, Zheng-hong QIN² \\ ${ }^{1}$ Institute of Neurology, Ruijin Hospital, Shanghai Jiao Tong University School of Medicine, Shanghai 200025, China; ${ }^{2}$ Department \\ of Pharmacology and Laboratory of Aging and Nervous Diseases, Soochow University School of Pharmaceutical Science, Suzhou \\ 215123, China
}

Acta Pharmacologica Sinica (2013) 34: 583-584; doi: 10.1038/aps.2013.53

The word autophagy is derived from the Greek roots "auto"(self) and "phagy" (eating) and broadly refers to the cellular catabolic processes, in which cytoplasmic materials are transported to lysosomes for degradation. Generally, autophagy is divided into three categories: macroautophagy, microautophagy and chaperone-mediated autophagy (CMA). Started four decades ago, autophagy research has expanded from a relatively minor research area to one of the most exciting and important topic in cell biology. For example, the total number of autophagy papers published in peer review journals worldwide has been increased 15 fold in the past ten years, and many of them are present in the top journals such as Nature, Sciences and Cell. A first specific journal "Autophagy" emerged in 2002, has become one of the leading international journals in biology. Meanwhile, a number of autophagy-related genes and pathways have been identified and innovative tools for genetic manipulation and chemical compounds to target autophagy components and pathways have been developed ${ }^{[1-4]}$. Furthermore, a comprehensive methodology article "Guidelines for the use and interpretation of assays for monitoring autophagy" published in 2008 and revised in 2012 is very useful for investigators to conduct the research in the field of autophagy ${ }^{[5,6]}$. Moreover, databases that are specifically dedicated to autophagy can be found in the websites of Human Autophagy-dedicated Database (HADb; www.autophagy.lu) and the Autophagy Database (http://tp-apg.genes.nig.ac.jp/autophagy/).

In order to develop new concepts and strategies for investigating the autophagy biology and function, and uncovering its relevance with diseases, autophagy research needs highly collaborative and interactive efforts between the different disciplines and specific areas. Important progress has been achieved in the recent years in defining the role of autophagy in human diseases such as cancers, cardiovascular

\footnotetext{
* To whom correspondence should be addressed.

E-mail wdle@sibs.ac.cn
}

diseases, metabolism disorders, immune-mediated diseases, and neurodegenerative diseases, etc. In addition, a number of new drugs in the clinical trials against cancers, immune diseases, and neurodegenerative diseases affect or act through autophagy. It is conceivable that exploring the molecular mechanisms of autophagy opens an avenue for development of novel drugs to treat these devastating diseases.

In the recent years, Chinese researchers have contributed a great deal to autophagy research in the basic and clinical aspects $^{[7-11]}$, and published numerous articles that account for $10 \%$ the total worldwide in this topic, and the percentage is steadily increasing. By the way, "Autophagy: biology and disease", a monograph in Chinese, was published in 2011 ${ }^{[12]}$, and became one of the hot selling books in China in 2012. In order to give a broad picture on recent progress in autophagy research and help gain a deep insight into autophagy biology and the related disease, Acta Pharmacologica Sinica launched this special edition "Autophagy and Drug Discovery". As the editors for this special edition, we invited several experts in China and abroad to contribute 13 articles, which covered the important aspects of basic and clinical research on autophagy and refleced the updated development in this field. Several major issues in autophagy research were highlighted in this special edition:

1. Autophagy regulates important biological functions, such as cell survival, cell death, cell metabolism, development, aging, infection and immunity. At a cellular level, the involvement of autophagy in the cell death and cell survival process appears to be complex. The multifunctional roles of autophagy are explained by its ability to interact with distinct key components in various cell pathways.

2. Autophagy has its own regulatory mechanisms, but this process is not isolated. Autophagy is coordinated with other cellular activities to maintain cell homeostasis. Owing to the dual roles of autophagy in cell death and the specificity of disease progression, the exact roles and underlying mechanisms of autophagy in various diseases are not fully 
understood. The application of autophagy inhibitors and activators will help us understand the regulation of autophagy in human diseases and provide insight into the development of autophagy-targeted drugs.

3. Although cells can manifest a clear increase in the numbers of autophagosomes shortly before or during their death, this phenomenon is sometimes due to defects in autophagosomal maturation and, hence, decreased, rather than increased, autophagy. For many diseases, the upregulation of autophagy is a promising therapeutic target. Combining the knowledge of autophagy compromise in neurodegenerative deseases with that of signaling pathways and drugs available to control autophagy may promote the development of optimal therapeutics.

4. Dysfunctional autophagy is found in ageing tissues and several ageing-associated diseases. The lifespan of model organisms such as yeast, worms, flies, and mice can be extended through promoting autophagy, either by genetic manipulations such as over-expression of Sirtuin 1, or by administrations of rapamycin, resveratrol or spermidine, supporting the notion that autophagy may play an important role in delaying ageing or extending lifespan.

5. Cells deploy two mechanisms for intracellular protein degradation: the ubiquitin-proteasome system and the autophagy-lysosome system. The UPS and autophagy have long been viewed as independent and parallel degradation systems with no point of intersection. Several lines of evidence have suggested that the UPS and autophagy are functionally interrelated catabolic processes.

6. Impaired autophagic flux has been linked to a variety of human pathophysiological process, including neurodegeneration, cancer, myopathy, cardiovascular and immunemediated disorders. There is a growing need to identify and quantify the status of autophagic flux in different pathological conditions. Given that autophagy is a highly dynamic and complex process regulated at multiple steps, it is of importance to measure the rate of delivery of autophagy substrates to lysosomes or the vacuole, followed by degradation, rather than to assess the steady-state levels of autophagosomes in an isolated approach (eg, by counting GFP-LC3 puncta, monitoring the amount of LC3-II without examining turnover, or by single time point electron micrographs) ${ }^{[6]}$. It is strongly recommended to use multiple assays whenever possible, instead of relying on the results from a single method to assess autophagic flux.

7. Encouraging progress has been made in autophagy-based anticancer therapeutics, and some autophagy-modulating drugs such as rapamycin and tamoxifen have been approved for clinical use in cancer management, and thus convincing autophagy as a promising therapeutic target in cancer. More importantly, attractive strategies for the identification of autophagy-modulating agents are emerging, which may undoubtedly provide new clues for launching a new wave in anticancer drug discovery. A better understanding of potential molecular mechanisms implicated in autophagy and the intrinsic relationships between autophagy and cancers at the level of entire network will help achieve the ultimate goal of conquering cancers.

Owing to the participation of more and more researchers from various fields and increasing funding opportunity, we will see continuous expansion and progress in autophagy research. It may be particularly interest to study the interactions of autophagy within the subgroup of autophagy family in mammals cells such as aggephagy, CMA, lippophagy, mitophagy, pexophagy, reticulophagy, ribophagy, xenophagy, and zymophagy/crinophagy ${ }^{[1]}$, to define the roles of other cellular activity in macromolecular degradation and cell metabolism; to study the underlying mechanisms of dual effects of autophagy on cell survival; to determine if selective manipulation of autophagy pathway could be a therapeutic strategy for cancers, metabolic disorders, immune diseases, and neurodegenerative disorders. We expect that a better understanding the roles and regulation of autophagy could bring us deeper insight into pathogenic mechanisms of many diseases and provide useful information leading to development of novel therapies.

\section{References}

1 Jin M, Liu X, Klionsky DJ. SnapShot: Selective Autophagy. Cell 2013; 152: 368-9.

2 Klionsky DJ, Baehrecke EH, Brumell JH, Chu CT, Codogno P, Cuervo $\mathrm{AM}$, et al. A comprehensive glossary of autophagy-related molecules and processes (2nd edition). Autophagy 2011; 7: 1273-94.

3 Mizushima N, Yoshimori T, Levine B. Methods in mammalian autophagy research. Cell 2010; 140: 313-26.

4 Rubinsztein DC, Cuervo AM, Ravikumar B, Sarkar S, Korolchuk V, Kaushik S, et al. In search of an "autophagomometer". Autophagy 2009; 5: 585-9.

5 Klionsky DJ, Abeliovich H, Agostinis P, Agrawal DK, Aliev G, Askew DS, et al. Guidelines for the use and interpretation of assays for monitoring autophagy. Autophagy 2008; 16: 151-75.

6 Klionsky DJ, Abdalla F, Abeliovich H, Abraham RT, Acevedo-Arozena A, Adeli $\mathrm{K}$, et al. Guidelines for the use and interpretation of assays for monitoring autophagy. Autophagy 2012; 8: 445-544.

7 Du Y, Yang D, Li L, Luo G, Li T, Fan X, et al. An insight into the mechanistic role of p53-mediated autophagy induction in response to proteasomal inhibition-induced neurotoxicity. Autophagy 2009; 5: 663-75.

8 Zhang X, Li L, Chen S, Yang D, Wang Y, Zhang X, et al. Rapamycin treatment augments motor neuron degeneration in SOD1 (G93A) mouse model of amyotrophic lateral sclerosis. Autophagy 2011; 7 : 412-25.

9 Yi C, Ma M, Ran L, Zheng J, Tong J, Zhu J, et al. Function and molecular mechanism of acetylation in autophagy regulation. Science 2012; 27: 474-7.

10 Zhang Y, Yan L, Zhou Z, Yang P, Tian E, Zhang K, et al. SEPA-1 mediates the specific recognition and degradation of $P$ granule components by autophagy in C. elegans. Cell 2009; 136: 308-21.

11 Sheng R, Liu XQ, Zhang LS, Gao B, Han R, Wu YQ, et al. Autophagy regulates endoplasmic reticulum stress in ischemic preconditioning. Autophagy 2012; 8: 310-25.

12 Qin ZH, Le WD. Autophagy: biology and diseases. Beijing: Science Press; 2011. 